\title{
ANTIOXIDATIVE EFFECT OF EPIGALLOCATECHIN GALLATE AGAINST D-GALACTOSAMINE-INDUCED INJURY IN PRIMARY CULTURE OF RAT HEPATOCYTES
}

\author{
Alena Moravcová, Zuzana Červinková, Otto Kučera, Vojtěch Mezera, Halka Lotková
}

\author{
Charles University in Prague, Faculty of Medicine in Hradec Králové, Czech Republic: Department of Physiology
}

\begin{abstract}
Summary: Literature data support that green tea and its major component epigallocatechin gallate (EGCG) have powerful antioxidant effects. Contrary, hepatotoxicity can be induced by high-dose EGCG. The timing of exposure to green tea in relation to administration of hepatotoxic agent plays an import role too. The aim of our work was a verification of antioxidative effect of EGCG on D-galactosamine-induced injury in primary culture of rat hepatocytes. Hepatocytes were incubated with EGCG at concentrations of 1.25-10 $\mu \mathrm{M}$ and toxic D-galactosamine (GalN) for 24 hrs. Alternatively, hepatocytes were pretreated with EGCG for 24 hrs, and then incubated with EGCG and GalN for further 24 hrs. Cytotoxicity was analysed by lactate dehydrogenase activity, functional capacity by albumin production. Oxidative stress was evaluated from a production of malondialdehyde and glutathione content in the cells. EGCG protected hepatocytes against GalN-induced cytotoxicity but preventive treatment of intact hepatocytes with EGCG was required to diminish the development of hepatocyte injury. Oxidative stress induced in our study seems to overcome the ability of hepatocytes to improve GSH depletion and albumin production. Prolongation of the pretreatment with EGCG could be a promising strategy leading to amelioration of its hepatoprotective effect.
\end{abstract}

Key words: Epigallocatechin gallate; Hepatocytes; D-galactosamine; Oxidative stress

\section{Introduction}

Literature data indicate that green tea possesses a wide range of health-promoting effects including anti-cancer, anti-atherosclerotic, anti-diabetic, hypocholesterolemic or hypoglycemic activity (1-4). The main chemical components of unfermented green tea are polyphenols, the major ones are catechins, mainly epigallocatechin gallate (EGCG) which have powerful antioxidant effects. These compounds may be especially effective in preventing oxidative stress-related diseases (5). Liver injury is frequently associated with oxidative stress that plays a relevant role in the induction of cell death in hepatocytes. Therefore, antioxidative therapy represents a potential strategy to prevent liver injury. Experimental results support that oral treatment with green tea extracts can ameliorate a hepatotoxicity $(6,7)$. On the other side, there is a rising evidence that hepatotoxicity associated with increased lipid peroxidation can be induced by high-dose EGCG $(8,9)$. The timing of exposure to green tea in relation to administration of hepatotoxic agent plays an import role too (10).

D-galactosamine (GalN) is a highly selective hepatotoxin frequently used in experiments. GalN induces oxidative stress, apoptosis and necrosis in both liver tissue and cultured hepatocytes (11-13). In vivo GalN causes diffuse liver damage resembling viral hepatitis (14) thus contributing to the popularity of this experimental hepatotoxin.
Pharmacokinetic parameters of catechins are intensively studied. Intestine can play an important role in their low bioavailability how the catechins are conjugated here. Nevertheless, there are mostly the free catechins that seem to enter the tissues. In vitro model allow to eliminate the problem of low bioavaibility and to identify easily the concentrations of the tested substance that are primarily nontoxic to hepatocytes. The aim of our work was a verification of potential antioxidative effect of the major catechin in green tea (EGCG) on GalN-induced injury in primary culture of rat hepatocytes. Moreover, two strategies were confronted: hepatocytes treated only simultaneously with EGCG and D-GalN or additionally pretreated with EGCG.

\section{Materials and Methods}

\section{Chemicals}

Medium William's E without phenol red, fetal bovine serum, penicillin, streptomycin and glutamine were purchased from Pan Biotech GmbH (Germany), D-galactosamine, type I collagen, trypan blue, epigallocatechin gallate from Sigma-Aldrich. Kit for lactate dehydrogenase (DiaSys, Germany), collagenase (Collagenase NB 4 Standard Grade from Clostridium histolyticum, Biotech, Czech Republic), insulin (Actrapid, Hoechst, Germany), glucagon (Glucagen, Novo Nordisk, Germany), prednisolon (Solu-Decortin, Merck, 
USA), Rat Albumin Elisa Quantification Kit (Bethyl Lab. Inc., USA), and Cell Proliferation Reagent WST-1 (Roche, Germany) from suppliers mentioned in brackets.

\section{Hepatocyte isolation, cultivation and treatment}

All animal protocols were in full compliance with the guidelines for animal care approved by the Institutional Animal Use and Care Committee of the Charles University Prague, Czech Republic. Hepatocytes were isolated from male Wistar rats by collagenase perfusion (15). The viability of freshly isolated cells was more than $90 \%$ as confirmed by trypan blue exclusion test. Isolated hepatocytes were suspended in William's E medium supplemented with fetal bovine serum $(6 \%)$, glutamine $(2 \mathrm{mM})$, penicillin $(100 \mathrm{IU} / \mathrm{ml})$, streptomycin $(10 \mathrm{mg} / \mathrm{ml})$, insulin $(0.08 \mathrm{IU} / \mathrm{ml})$, prednisolon $(0.05 \mu \mathrm{g} / \mathrm{ml})$, glucagon $(0.008 \mu \mathrm{g} / \mathrm{ml})$ and plated in collagen-coated 24-well plates at a density of $70,000 / \mathrm{cm}^{2}$. Hepatocytes were allowed to attach in a gassed atmosphere $\left(5 \% \mathrm{CO}_{2}\right)$ at $37{ }^{\circ} \mathrm{C}$ for $2 \mathrm{~h}$. After the establishment of monolayers, the medium was removed and replaced with a fresh supplemented medium without fetal bovine serum containing dissolved epigallocatechin gallate at different concentrations of $1.25,2.5,5$ and $10 \mu \mathrm{M}$ and toxic substance D-galactosamine (GalN, $40 \mathrm{mM})$. The treatment period lasted $24 \mathrm{hrs}$ and then all medium was removed. Alternatively, hepatocytes were pretreated with EGCG for $24 \mathrm{hrs}$, and then the medium was replaced by medium with EGCG and toxic GalN for further $24 \mathrm{~h}$. At the end of incubation period, the medium was collected for biochemical assays.

\section{Biochemical assays}

Hepatocyte toxicity was determined by the activity of lactate dehydrogenase (LDH) leakage. LDH activity in the culture medium and in lysate of hepatocytes (frozen and lysed cells in distilled water) was measured using a commercial kit. Concentration of malondialdehyde (MDA) was measured as TBARS in the culture medium (25) and served as a marker of lipid peroxidation. To evaluate the functional capacity of cultured hepatocytes the amount of albumin secreted into the culture medium during the incubation was measured using the ELISA kit. The nontoxic effect of EGCG on hepatocyte culture was tested by a colorimetric assay based on the cleavage of the tetrazolium salt WST-1 by mitochondrial dehydrogenases in viable cells. After cell lysis in a freezer $\left(-80^{\circ} \mathrm{C}, 10 \mathrm{~min}\right)$ and the harvesting of hepatocytes, glutathione content was determined by the spectrofluorometric assay (16).

\section{Statistical analysis}

Experiments were repeated at least three times using different isolations of hepatocytes. The results are expressed as a mean \pm SD. Statistical significance was analysed using the one-way ANOVA test followed by Dunnett's post hoc test (GraphPad Prism 6 for Windows, Graphpad Software, USA). $\mathrm{p}<0.05$ was considered to be statistically significant.

\section{Results}

Nontoxic effect of epigallocatechin gallate (EGCG) at the doses of $1.25,2.5,5$ and $10 \mu \mathrm{mol} / \mathrm{l}$ was documented by WST-1 assay (Fig. 1). Concentrations from $20 \mu \mathrm{M}$ were toxic.

The cytotoxicity induced in hepatocyte culture was investigated by measuring of LDH leakage (Fig. 2). D-galactosamine (GalN) caused significant increase in LDH leakage

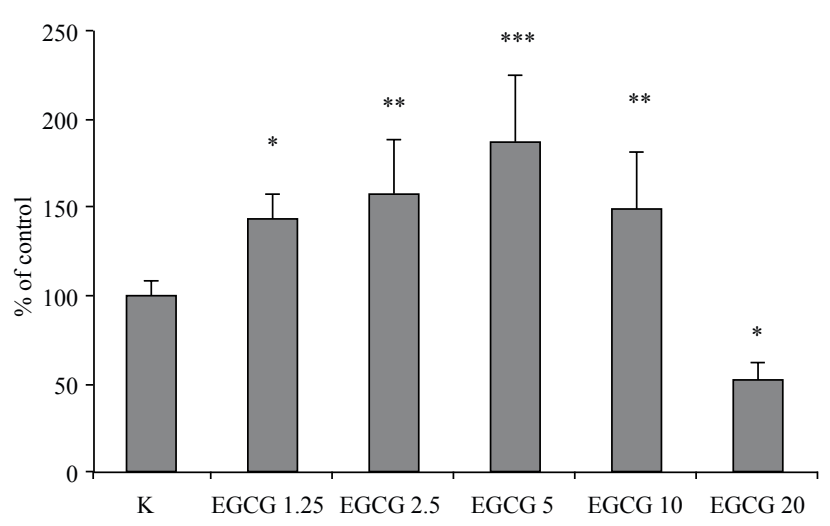

Fig. 1: Mitochondrial dehydrogenase activity of intact hepatocytes measured with WST-1 assay after 24 hours exposure to different concentrations of epigallocatechin gallate (EGCG) - 1.25; 2.5; $5 ; 10$ and $20 \mu \mathrm{mol} / \mathrm{l}$. WST of control hepatocytes is expressed as $100 \%$. Each column represents the mean value and bars the S.D. $(\mathrm{n}=8) * \mathrm{p}<0.05, * * \mathrm{p}<0.01, * * * \mathrm{p}<0.001$ difference from untreated control $\mathrm{K}$.

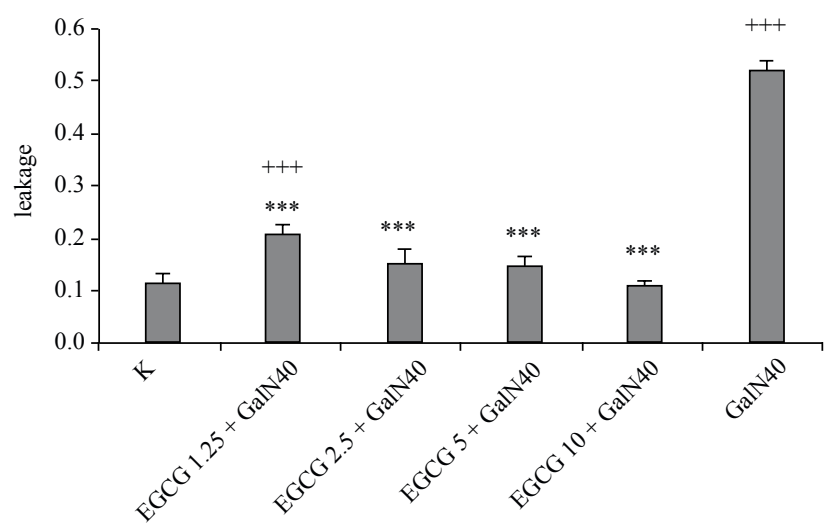

Fig. 2: Lactate dehydrogenase (LDH) leakage (the ratio of LDH activity in the medium and in the medium plus in the hepatocytes) after the pretreatment with epigallocatechin gallate (EGCG) $1.25 ; 2.5 ; 5$ and $10 \mu \mathrm{mol} / 1$ for 24 hours and further incubation with EGCG $(1.25,2.5,5,10 \mu \mathrm{M})$ and D-galactosamine $(40 \mathrm{mM})$. Each column represents the mean value and bars the S.D. $(n=6)$. $+++\mathrm{p}<0.001$ difference from $\mathrm{K}, * * * \mathrm{p}<0.001$ difference from GalN40. K: untreated control, GalN40: cultures treated with $40 \mathrm{mM}$ D-galactosamine. 
in comparison with control group $(\mathrm{p}<0.001)$. This increase was prevented only when hepatocytes were preincubated with EGCG. EGCG significantly decreased LDH leakage at all concentrations we used $(\mathrm{p}<0.001)$. However, simultaneous treatment of hepatocytes with EGCG and GalN did not protect LDH leakage. Therefore, LDH as well as markers of oxidative stress and functional parametres from the simultaneous treatment are not included in the results.

GalN induced a progressive increase in concentration of malondialdehyde (MDA, $\mathrm{p}<0.001$ ) which served as

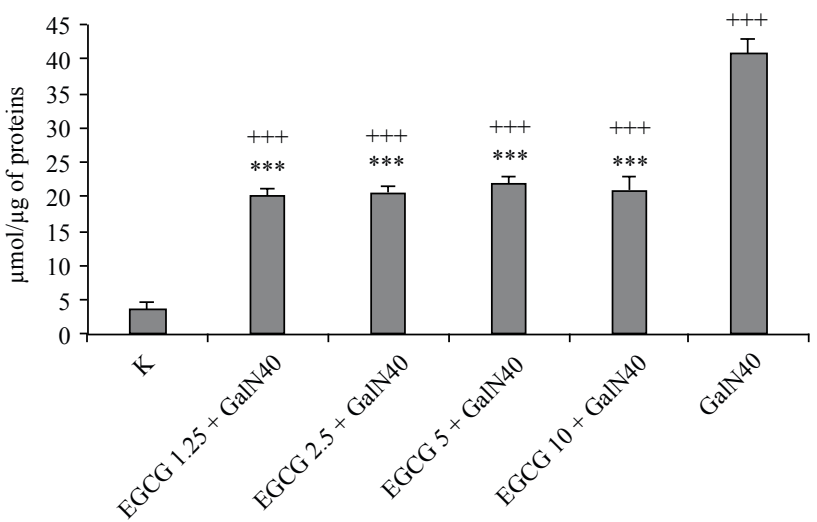

Fig. 3: Malondialdehyde production by hepatocytes after the pretreatment with epigallocatechin gallate $(\mathrm{EGCG})-1.25 ; 2.5 ; 5$ and $10 \mu \mathrm{mol} / 1$ for 24 hours and further incubation with EGCG $(1.25,2.5$, $5,10 \mu \mathrm{M})$ and D-galactosamine $(40 \mathrm{mM})$. Each column represents the mean value and bars the S.D. $(n=6)$. $+++p<0.001$ difference from $\mathrm{K},{ }^{* * *} \mathrm{p}<0.001$ difference from GalN40. K: untreated control, GalN40: cultures treated with $40 \mathrm{mM}$ D-galactosamine.

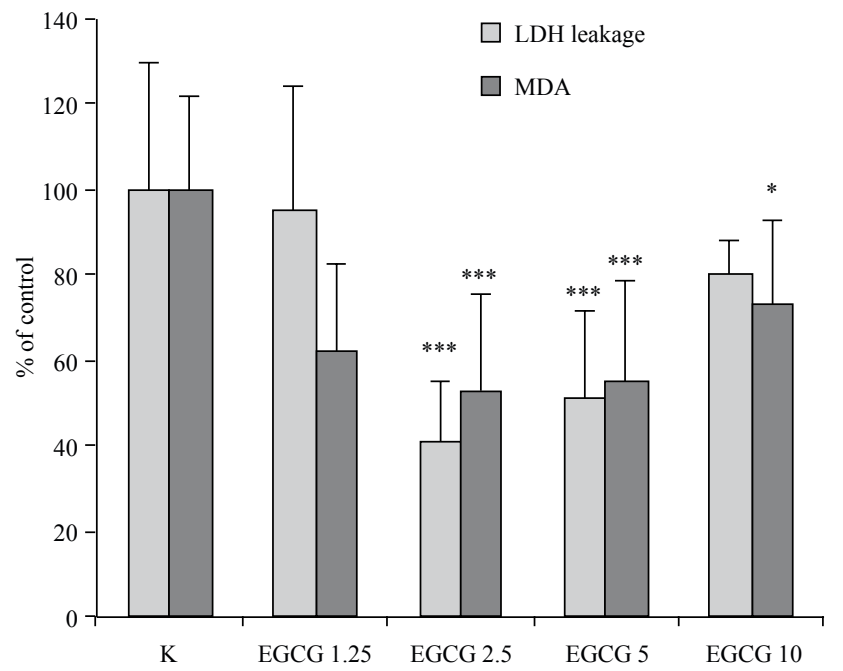

Fig. 4: Effect of epigallocatechin gallate (EGCG) $-1.25 ; 2.5 ; 5$ and $10 \mu \mathrm{mol} / 1$ on lactate dehydrogenase (LDH) leakage and malondialdehyde (MDA) production from intact hepatocytes. LDH leakage and MDA production of control hepatocytes is expresses as $100 \%$. Each column represents the mean value and bars the S.D. $(n=6)$. ${ }^{*} \mathrm{p}<0.05, * * * \mathrm{p}<0.001$ difference from untreated control K. a marker of lipid peroxidation (Fig. 3). EGCG prevented GalN-induced lipid peroxidation in the same extent at all concentrations we used ( $p<0.001)$. Fig. 4 documents that EGCG alone did not impair the integrity of hepatocyte cell membrane and did not increase the lipid peroxidation.

GalN alone caused a significant decrease in glutathione content (GSH+GSSG, $\mathrm{p}<0.001)$. Glutathione content was not protected by the pretreatment with EGCG (Fig. 5). Never-

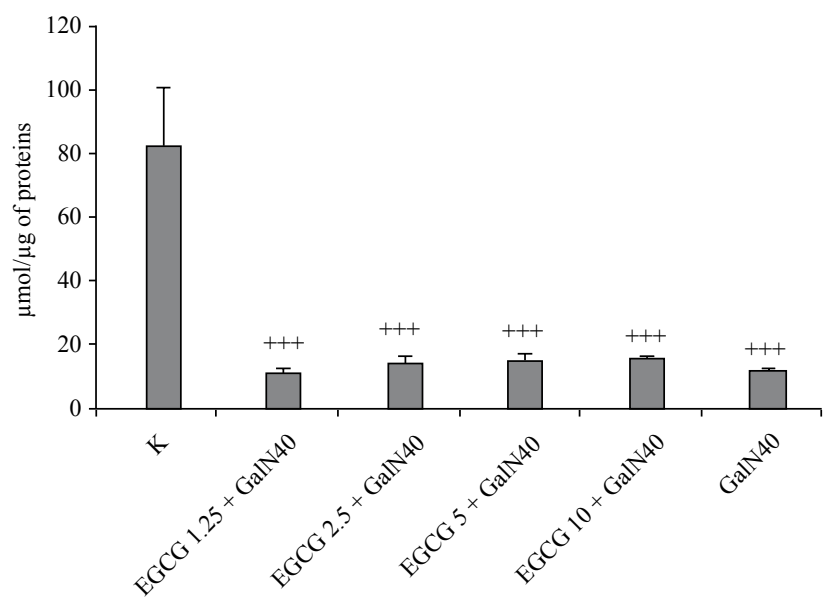

Fig. 5: Total content of glutathione (GSH+ GSSG) in hepatocytes after the pretreatment with epigallocatechin gallate (EGCG) $1.25 ; 2.5 ; 5$ and $10 \mu \mathrm{mol} / 1$ for 24 hours and further incubation with EGCG $(1.25,2.5,5,10 \mu \mathrm{M})$ and D-galactosamine $(40 \mathrm{mM})$. Each column represents the mean value and bars the S.D. $(n=6)$. $+++p<0.001$ difference from K. K: untreated control, GalN40: cultures treated with $40 \mathrm{mM}$ D-galactosamine.

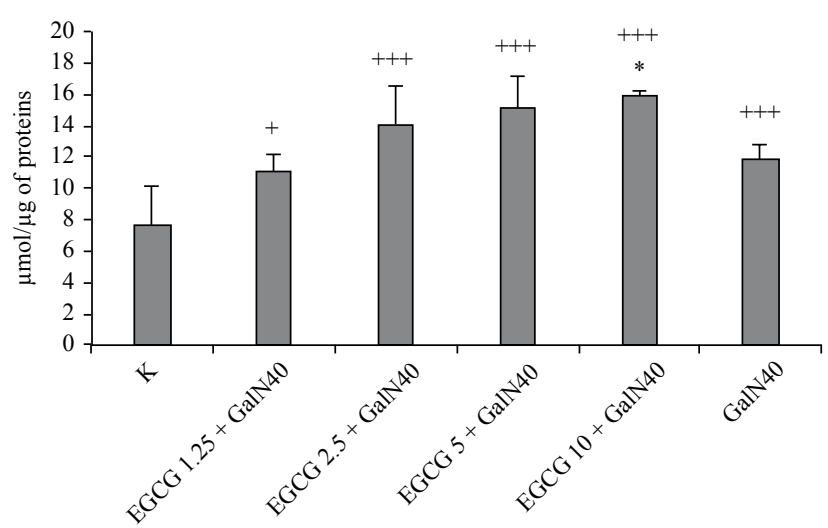

Fig. 6: Content of oxidized form of glutathione (GSSG) in hepatocytes after the pretreatment with epigallocatechin gallate (EGCG) $-1.25 ; 2.5 ; 5$ and $10 \mu \mathrm{mol} / 1$ for 24 hours and further incubation with EGCG $(1.25,2.5,5,10 \mu \mathrm{M})$ and D-galactosamine $(40 \mathrm{mM})$. Each column represents the mean value and bars the S.D. $(\mathrm{n}=6)$. $+p<0.05,+++p<0.001$ difference from $\mathrm{K}, * \mathrm{p}<0.05$ difference from GalN40. K: untreated control, GalN40: cultures treated with $40 \mathrm{mM}$ D-galactosamine. 


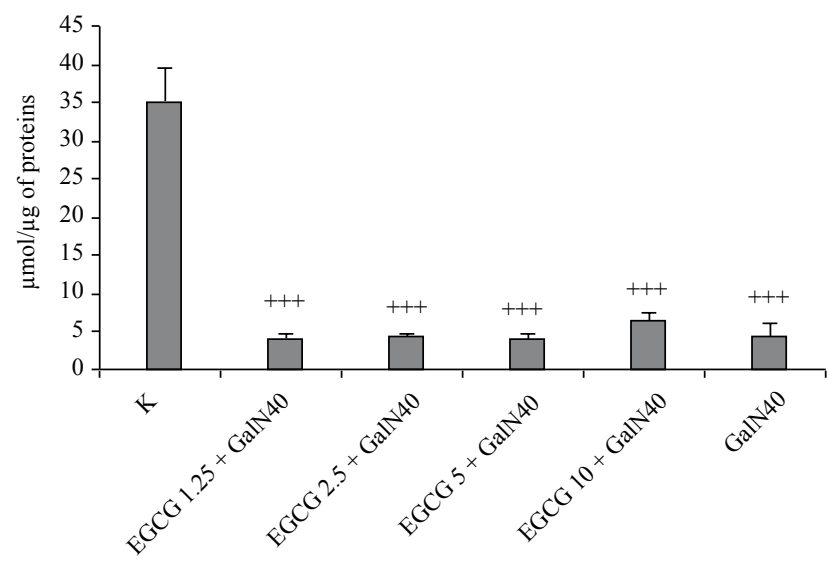

Fig. 7: Albumin production by hepatocytes after the pretreatment with epigallocatechin gallate (EGCG) $-1.25 ; 2.5 ; 5$ and $10 \mu \mathrm{mol} / 1$ for 24 hours and further incubation with EGCG $(1.25,2.5,5$, $10 \mu \mathrm{M})$ and D-galactosamine $(40 \mathrm{mM})$. Each column represents the mean value and bars the S.D. $(n=6) .+++p<0.001$ difference from K. K: untreated control, GalN40: cultures treated with $40 \mathrm{mM}$ D-galactosamine.

theless, GSSG significantly raised after the pretreatment with EGCG (Fig. 6) at the concentration of $10 \mu \mathrm{mol} / \mathrm{l}(\mathrm{p}<0.05)$.

Concentration of albumin in culture medium served as a marker of functional capacity of hepatocytes. Albumin production by hepatocytes decreased after the incubation with GalN ( $p<0.001)$. EGCG did not affect the functional capacity of hepatocytes at any concentration that we tested (Fig. 7).

\section{Discussion}

Flavonoids in green tea extracts have been found to play an important role in the protection against oxidative stress (17-19). As mentioned in Introduction antioxidative therapy represents a potential strategy to prevent liver injury. Literature data support the green tea and its major component EGCG as promising hepatoprotective agents. Nevertheless, an increasing evidence suggests that polyphenols can also behave as pro-oxidative agents leading to toxicity. Hepatotoxicity can be a consequence of high doses of green tea extracts $(8,9,20)$ or by the time at which the treatment occurs. Green tea extract administered to mice prior to hepatotoxic dose of acetaminophen (APAP) attenuated the hepatotoxicity whereas; the opposite develops when green tea extract was administered after APAP (10). These effects have been mainly tested on animals after intragastric administration. There is a free EGCG that was determined in the tissues after both intragastric and intravenous applications. Passing the intestine, EGCG is largely conjugated in the plasma contrary to i.v. administration when tissues can be exposed to higher concentration of the free EGCG. The free EGCG seems to enter the tissues more readily. Moreover, the lack of conjugated EGCG in the tissues may probably be due to its exclusion (21). Finally, the concentrations of EGCG in the tissues after intragastric treatment are lower than is expected from the plasma concentration. A relationship between dose and the percentage of conjugated versus unconjugated EGCG was not apparent (22). Therefore in vitro studies can better identify a dose of EGCG that promotes protective or toxic effect on hepatocytes. We have found that EGCG at concentrations of $1.25-10 \mu \mathrm{M}$ are nontoxic to the intact rat hepatocytes in primary culture. Cytotoxic effect was exerted by concentrations from $20 \mu \mathrm{M}$. Cytotoxicity tested in vitro by other authors on hepalc1c mouse hepatocytes was pronounced dose dependently from $100 \mu \mathrm{M}$ EGCG (8). Our work identifies that nontoxic 1.25-10 $\mu \mathrm{M}$ EGCG can prevent hepatocytes from D-galactosamineinduced cytotoxicity. An achievement of this protective effect depended on timing of EGCG treatment in relation to the administration of GalN. Preventive treatment of intact hepatocytes with EGCG was required to diminish the development of hepatocyte injury induced by GalN while only simultaneous treatment of hepatocytes with EGCG and GalN failed.

GalN-induced cell death is related to a rise of oxidative stress, increased production of reactive oxygen species (ROS) has been reported in vivo $(23,24)$ and in vitro (11, 13). In our experiment, the pretreatment of hepatocyte culture with EGCG protected a cell viability that paralleled an attenuation of lipid peroxidation induced by GalN. Catechins are effective scavengers. EGCG may decrease the concentration of lipid free radicals thus it may terminate the initiation and propagation of lipid peroxidation. When EGCG penetrates the lipid bilayer this protective effect can develop in the cytoplasmic membrane (25). Besides the induction of lipid peroxidation, GalN decreases GSH content in hepatocytes $(12,26)$. The cells respond to lipid peroxidation by antioxidant induction (27). GSH is an important protective molecule that acts as a free radical scavenger in the detoxification of reactive metabolites and ROS (10). There was documented that EGCG is preferentially used as an antioxidant, rather than GSH, to suppress lipid peroxidation and to protect cells from a decrease in GSH and oxidative damage $(5,28)$. In our study, while EGCG acts as an effective inhibitor of lipid peroxidation, decrease in total glutathione content (reduced, GSH, and oxidized, GSSG, forms) as well as its reduced form - GSH - was not protected. Nevertheless, we confirmed higher content of GSSG in hepatocytes pretreated with EGCG and further incubated with EGCG and hepatotoxic GalN. Green tea may increase GSH biosynthesis (29). We cannot exclude that intracellular GSH is more produced during our pretreatment of the intact hepatocytes with EGCG. Oxidative stress induced by GalN then can lead to oxidation of GSH in a higher extent in hepatocytes pretreated with EGCG. The oxidative stress may overcome the ability of the cells to reduce GSSG to GSH, leading to accumulation of GSSG within the cytosol (30). GSSG may also release the cell into medium (31) where the 
concentration of GSH and GSSG were not measured in our work. Moreover, EGCG has been reported to react with GSH in isolated hepatocytes and to cause a transient decrease in GSH $(10,32)$. Then simultaneous cultivation with GalN (without preceding pretreatment with EGCG) could fail as the decrease in GSH stores deepens.

GalN also influences metabolic functions. In our conditions, GalN suppressed albumin synthesis. The toxicity of GalN is related to the depletion of uridine pools and alteration of RNA and protein synthesis (14). EGCG is not known to influence the content of uridine in hepatocytes. The oxidative stress induced by GalN is accompanied by activation of proinflammatory cytokines (23). These cytokines can cause dramatic alterations in hepatic gene expression further followed by decreased albumin synthesis (33-35). Therefore, we cannot exclude that in our study, the albumin production attenuated in response to the proinflammatory cytokines. However, literature data indicate protective effect of the green tea on release and action of proinflammatory cytokines $(6,23,36)$ resulting in a reduction of hepatotoxicity, our finding did not confirmed the protective effect of EGCG on the albumin synthesis.

\section{Conclusion}

Only preventive treatment with EGCG protected rat hepatocytes from GalN induced toxicity in primary culture while EGCG given simultaneously with hepatotoxin failed. The reduction of lipid peroxidation can contribute to the protection against disruption of the cellular membrane. In spite of the effect of EGCG on GSH production cannot be excluded, the oxidative stress induced in our study seems to overcome the ability of hepatocytes to improve GSH depletion. This can result to the finding that the functional capacity of hepatocytes was not protected satisfactory. Prolongation of the pretreatment with EGCG could be a promising strategy leading to amelioration of its hepatoprotective effect.

\section{Acknowledgements}

This work was supported by grants PRVOUK P37/02 and SVV-260058.

\section{References}

1. Cuccioloni M, Mozzicafreddo M, Spina M, et al. Epigallocatechin-3-gallate potently inhibits the in vitro activity of hydroxy-3-methyl-glutaryl-CoA reductase. J Lipid Res 2011; 52: 897-907.

2. Liu K, Zhou R, Wang B, et al. Effect of green tea on glucose control and insulin sensitivity: a meta-analysis of 17 randomized controlled trials. Am J Clin Nutr 2013; 98: $340-8$

3. Sone T, Kuriyama S, Nakaya N, et al. Randomized controlled trial for an effect of catechin-enriched green tea consumption on adiponectin and cardiovascular disease risk factors. Food Nutr Res 2011; doi: 10.3402/fnr.v55i0.8326.

4. Yu Y, Deng Y, Lu BM, Liu YX, Li J, Bao JK. Green tea catechins: a fresh flavor to anticancer therapy. Apoptosis 2013; 19: 1-18

5. Jimenez-Lopez JM, Cederbaum AI. Green tea polyphenol epigallocatechin-3-gallate protects HepG2 cells against CYP2E1-dependent toxicity. Free Radic Bio Med 2004; 36: 359-70.
6. He P, Noda Y, Sugiyama K. Green tea suppresses lipopolysaccharide-induced liver injury in d-galactosamine-sensitized rats. J Nutr 2001; 131: 1560-7.

7. Mahmoud MF, Fahmy A, Auf MA. Evaluation of the hepatoprotective effect of green tea extract and selenium on CCL4-induced fibrosis. e-SPEN Journal 2012; 7: e23-e9.

8. Inoue H, Maeda-Yamamoto M, Nesumi A, Murakami A. Delphinidin-3-O-galactoside protects mouse hepatocytes from (-)-epigallocatechin-3-gallate-induced cytotoxicity via up-regulation of heme oxygenase-1 and heat shock protein 70 . Nutr Res 2012; 32: 357-64.

9. Lambert JD, Kennett MJ, Sang S, Reuhl KR, Ju J, Yang CS. Hepatotoxicity of high oral dose (-)-epigallocatechin-3-gallate in mice. Food Chem Toxicol 2010; 48: 409-16.

10. Lu Y, Sun J, Petrova K, et al. Metabolomics evaluation of the effects of green tea extract on acetaminophen-induced hepatotoxicity in mice. Food Chem Toxicol 2013; 62: 707-21.

11. Gonzalez R, Ferrin G, Hidalgo AB, et al. N-acetylcysteine, coenzyme Q10 and superoxide dismutase mimetic prevent mitochondrial cell dysfunction and cell death induced by d-galactosamine in primary culture of human hepatocytes. Chem-Biol Interact 2009; 181: 95-106.

12. Kucera O, Cervinkova Z, Lotkova H, et al. Protective effect of S-adenosylmethionine against galactosamine-induced injury of rat hepatocytes in primary culture. Physiol Res 2006; 55: 551-60.

13. Quintero A, Pedraza CA, Siendones E, et al. PGE1 protection against apoptosis induced by D-galactosamine is not related to the modulation of intracellular free radical production in primary culture of rat hepatocytes. Free Radic Res 2002; 36: $345-55$

14. Keppler D RJ, Bischoff E, Decker K. The trapping of uridine phosphates by D-galactosamine, D-glucosamine and 2-deoxy-D-galactose. A study of the mechanism of galactosamine hepatitis. Eur J Biochem 1970; 17: 246-53.

15. Berry MN, Edwards AM, Barritt GJ. Isolated Hepatocytes Preparation, Properties and Application. In: Biochemistry and Molecular Biology (Burdon, RH, van Knippenberg, PH, eds), New York, Oxford: Elsevier Amsterdam, 1991: 18-81.

16. Rousar T, Kucera O, Lotkova H, Cervinkova Z. Assessment of reduced glutathione: comparison of an optimized fluorometric assay with enzymatic recycling method. Anal Biochem 2012; 423: 236-40.

17. Babich H, Gold T, Gold R. Mediation of the in vitro cytotoxicity of green and black tea polyphenols by cobalt chloride. Toxicol Lett 2005; 155: 195-205.

18. El-Beshbishy HA. Hepatoprotective effect of green tea (Camellia sinensis) extract against tamoxifen-induced liver injury in rats. J Biochem Mol Biol 2005; 38: 563-70.

19. Okada K, Wangpoengtrakul C, Tanaka T, Toyokuni S, Uchida K, Osawa T. Curcumin and especially tetrahydrocurcumin ameliorate oxidative stress-induced renal injury in mice. J Nutr 2001; 131: 2090-5.

20. Isbrucker RA, Edwards JA, Wolz E, Davidovich A, Bausch J. Safety studies on epigallocatechin gallate (EGCG) preparations. Part 2: dermal, acute and short-term toxicity studies. Food Chem Toxicol 2006; 44: 636-50.

21. Lambert JD, Lee MJ, Lu H, et al. Epigallocatechin-3-gallate is absorbed but extensively glucuronidated following oral administration to mice. J Nutr 2003; 133: $4172-7$.

22. Lambert JD, Lee MJ, Diamond L, et al. Dose-dependent levels of epigallocatechin3-gallate in human colon cancer cells and mouse plasma and tissues. Drug Metab Dispos 2006; 34: 8-11.

23. Lin BR, Yu CJ, Chen WC, et al. Green tea extract supplement reduces D-galactosamine-induced acute liver injury by inhibition of apoptotic and proinflammatory signaling. J Biomed Sci 2009; 16: 35.

24. Yoshikawa T, Furukawa Y, Murakami M, Takemura S, Kondo M. Effects of vitamin E on D-galactosamine-induced or carbon tetrachloride-induced hepatotoxicity. Digestion 1982; 25: 222-9.

25. Saffari Y, Sadrzadeh SM. Green tea metabolite EGCG protects membranes against oxidative damage in vitro. Life Sci 2004; 74: 1513-8.

26. McMillan JM, Jollow DJ. Galactosamine hepatotoxicity: effect of galactosamine on glutathione resynthesis in rat primary hepatocyte cultures. Toxicol Appl Pharm 1992; 115: $234-40$

27. Seven A, Guzel S, Seymen O, et al. Effects of vitamin E supplementation on oxidative stress in streptozotocin induced diabetic rats: investigation of liver and plasma. Yonsei Med J 2004; 45: 703-10.

28. Murakami C, Hirakawa Y, Inui H, Nakano Y, Yoshida H. Effects of epigallocatechin 3-O-gallate on cellular antioxidative system in HepG2 cells. J Nutr Sci Vitaminol 2002; 48: 89-94.

29. Park HJ, DiNatale DA, Chung MY, et al. Green tea extract attenuates hepatic steatosis by decreasing adipose lipogenesis and enhancing hepatic antioxidant defenses in ob/ob mice. J Nutr Biochem 2011; 22: 393-400.

30. Lu SC. Regulation of hepatic glutathione synthesis: current concepts and controversies. FASEB J 1999; 13: 1169-83.

31. Lotkova H, Cervinkova Z, Kucera O, Krivakova P, Kandar R. Protective effect of S-adenosylmethionine on cellular and mitochondrial membranes of rat hepatocytes against tert-butylhydroperoxide-induced injury in primary culture. Chem Biol Interact 2005; 156: 13-23.

32. Galati G, Lin A, Sultan AM, O'Brien PJ. Cellular and in vivo hepatotoxicity caused by green tea phenolic acids and catechins. Free Rad Biol Med 2006; 40: 570-80. 
33. Marinkovic S, Jahreis GP, Wong GG, Baumann H. IL-6 modulates the synthesis of a specific set of acute phase plasma proteins in vivo. J Immunol 1989; 142: 808-12.

34. Sharma RJ, Macallan DC, Sedgwick P, Remick DG, Griffin GE. Kinetics of endotoxin-induced acute-phase protein gene expression and its modulation by TNF-alpha monoclonal antibody. Am J Physiol 1992; 262: R786-93.
35. Wang XY, Li WQ, Lu J, Li N, Li JS. Mechanism of reduction of albumin expression induced by lipopolysaccharide in rat hepatocytes. Chinese Med J 2005; 118 1695-702.

36. Abe K, Ijiri M, Suzuki T, Taguchi K, Koyama Y, Isemura M. Green tea with a high catechin content suppresses inflammatory cytokine expression in the galactosamine-injured rat liver. Biomed Res 2005; 26:187-92.

Received: $28 / 02 / 2014$

Corresponding author:

Halka Lotková, Department of Physiology, Charles University in Prague, Faculty of Medicine in Hradec Králové, Šimkova 870, 50038 Hradec Králové, Czech Republic; e-mail: lotko@1fhk.cuni.cz 